Mathematical Sciences and Applications

E-NOTES

MATHEMATICAL

SCIENCES

AND APPLICATIONS

E-NOTES

https://doi.org/10.36753/msaen.893469

10 (3) 125-134 (2022) - Research Article

ISSN: 2147-6268

(c)MSAEN

\title{
Gould-Hopper Based Degenerate Truncated Bernoulli Polynomials
}

\author{
Uğur Duran
}

\begin{abstract}
In this study, we consider Gould-Hopper based truncated degenerate Bernoulli polynomials and examine diverse properties and formulas covering addition formulas, correlations and derivation property. Then, we derive some interesting implicit summation formulas and symmetric identities. Moreover, we define Gould-Hopper based truncated degenerate Bernoulli polynomials of order $r$ and give some of their properties and relations.

Keywords: Degenerate exponential function; truncated exponential function; Bernoulli polynomials; Gould-Hopper polynomials; exponential generating function.
\end{abstract}

AMS Subject Classification (2020): Primary: 11B73; Secondary: 11B68; 33B10.

\section{Introduction}

Along this paper, the usual notations $\mathbb{N}, \mathbb{N}_{0}, \mathbb{R}$ and $\mathbb{C}$, are referred to the set of all natural numbers, the set of all non-negative integers, the set of all real numbers and the set of all complex numbers, respectively.

The truncated form of the exponential polynomials $e_{n}(z)$ are the first $(n+1)$ terms of the Taylor series for $e^{z}(c f$. [3]) at $z=0$, namely

$$
e_{n}(z)=\sum_{k=0}^{n} \frac{z^{k}}{k !}
$$

One can see [3] to get the detailed information about $e_{n}(z)$.

For $\lambda \in \mathbb{C}$, the $\lambda$-falling factorial $(z)_{n, \lambda}$ is defined by $(z)_{n, \lambda}=z(z-\lambda)(z-2 \lambda) \cdots(z-(n-1) \lambda)$ for $n \in \mathbb{N}$ with $(z)_{0, \lambda}=1, c f$. $[1,4,8,12]$. In the case $\lambda=1$, the $\lambda$-falling factorial becomes to the usual falling factorial given by $(z)_{n, 1}:=(z)_{n}=z(z-1) \cdots(z-n+1)$ with $(z)_{0,1}=1$.

Let $\lambda \in \mathbb{R} /\{0\}$. The degenerate form of the exponential function $e_{\lambda}^{z}(z)$ is defined by $(c f .[1,4,5,8,10-14])$

$$
e_{\lambda}^{\omega}(z)=(1+\lambda z)^{\frac{\omega}{\lambda}} \text { and } e_{\lambda}^{1}(z):=e_{\lambda}(z) .
$$


We note that $\lim _{\lambda \rightarrow 0} e_{\lambda}^{\omega}(z)=e^{\omega z}$. From (1.2), we attain

$$
e_{\lambda}^{\omega}(z)=\sum_{n=0}^{\infty}(\omega)_{n, \lambda} \frac{z^{n}}{n !}
$$

The degenerate truncated form of the exponential polynomials (also called the Detr-exponential polynomials) are considereed as the first $(n+1)$ terms of the Mac Laurin series expansion of $e_{\lambda}(z)$ in (1.3) (cf. [8]):

$$
e_{n, \lambda}(z)=\sum_{k=0}^{n}(1)_{k, \lambda} \frac{z^{k}}{k !}
$$

Also, when $\lambda \rightarrow 0$, the polynomials $e_{n, \lambda}(z)$ (1.4) become the polynomials $e_{n}(z)$ in (1.1). To get more detailed information about the Detr-exponential polynomials and their properties, see [8].

The Stirling numbers $S_{2}(n, k)$ and polynomials $S_{2}(n, k: \omega)$ of the second kind are provided as follows (cf. $[8,12,17]):$

$$
\sum_{n=0}^{\infty} S_{2}(n, k) \frac{z^{n}}{n !}=\frac{\left(e^{z}-1\right)^{k}}{k !} \text { and } \sum_{n=0}^{\infty} S_{2}(n, k: \omega) \frac{z^{n}}{n !}=\frac{\left(e^{z}-1\right)^{k}}{k !} e^{z \omega} .
$$

The degenerate form of the Stirling polynomials of the second kind are given below (cf. [6-8,11-13]):

$$
\sum_{n=0}^{\infty} S_{2, \lambda}(n, k: \omega) \frac{z^{n}}{n !}=\frac{\left(e_{\lambda}(z)-1\right)^{k}}{k !} e_{\lambda}^{\omega}(z) .
$$

The degenerate truncated form of the Stirling polynomials of the second kind are considered as follows (cf. [8]):

$$
\sum_{n=0}^{\infty} S_{2, m ; \lambda}(n, k: \omega) \frac{z^{n}}{n !}=\frac{\left(e_{\lambda}(z)-1-e_{m-1, \lambda}(z)\right)^{k}}{k !} e_{\lambda}^{\omega}(z)
$$

The Gould-Hopper polynomials $H_{n}^{(j)}(\omega, \theta)$ are defined by (see [4,6-8,15]):

$$
\sum_{n=0}^{\infty} H_{n}^{(j)}(\omega, \theta) \frac{z^{n}}{n !}=e^{\omega z+\theta z^{j}}
$$

where $j \in \mathbb{N}$ with $j \geq 2$. Choosing $j=1$ in (2.14), the polynomials $H_{n}^{(j)}(\omega, \theta)$ reduce to the Newton binomial formula. Moreover, taking $j=2$ in (2.14), the polynomials $H_{n}^{(j)}(\omega, \theta)$ become the Hermite polynomials $H_{n}(\omega, \theta)$ (cf. [15]). The two polynomials $H_{n}^{(j)}(\omega, \theta)$ and $H_{n}(\omega, \theta)$ have been utilized to generalize multifarious special polynomials including Bell, Bernoulli, Genocchi and Euler polynomials (see [4,6-8,15]).

Let $j \in \mathbb{N}$ and $\lambda \in \mathbb{R} \backslash\{0\}$. The degenerate Gould-Hopper polynomials $H_{n, \lambda}^{(j)}(\omega, \theta)$ are defined below $(c f$. [4,6,7]):

$$
\sum_{n=0}^{\infty} H_{n, \lambda}^{(j)}(\omega, \theta) \frac{z^{n}}{n !}=e_{\lambda}^{x}(z) e_{\lambda}^{y}\left(z^{j}\right) .
$$

Diverse applications and properties of the polynomials $H_{n, \lambda}^{(j)}(\omega, \theta)$ are investigated in $[4,6,7]$.

\section{The Gould-Hopper Based Degenerate Truncated Bernoulli Polynomials}

In this chapter, we consider the Gould-Hopper based degenerate truncated Bernoulli polynomials and examine diverse formulas and correlations such as implicit summation formulas, derivation rule and symmetric identities.

The Bernoulli polynomials are defined below (cf. [1,2,6-10,14,16,18]):

$$
\sum_{n=0}^{\infty} B_{n}(x) \frac{z^{n}}{n !}=\frac{z}{e^{z}-1} e^{x z} . \quad(|z|<2 \pi)
$$


The degenerate form of the Bernoulli polynomials are given below $(c f .[1,8,10,14])$ :

$$
\sum_{n=0}^{\infty} B_{n, \lambda}(x) \frac{z^{n}}{n !}=\frac{2 z}{e_{\lambda}(z)+1} e_{\lambda}^{x}(z) .
$$

The truncated form of the Bernoulli polynomials $B_{m, n}(x)$ are provided below (cf. [3,9]):

$$
\sum_{n=0}^{\infty} B_{m, n}(x) \frac{z^{n}}{n !}=\frac{\frac{z^{m+1}}{(m+1) !}}{e^{z}-1-e_{m-1}(z)} e^{x z} .
$$

Thanks to many mathematicians, recently, multifarious truncated and degenerate extensions of the Bernoulli polynomials have been considered and invesitgated in [1,4,6-10,14,16].

The degenerate truncated Bernoulli polynomials are defined below (cf. [8]):

$$
\sum_{n=0}^{\infty} B_{m, n, \lambda}(x) \frac{z^{n}}{n !}=\frac{(1)_{m+1, \lambda} \frac{z^{m+1}}{(m+1) !}}{e_{\lambda}(z)-1-e_{m-1, \lambda}(z)} e_{\lambda}^{x}(z) .
$$

When $x=0$, we have $B_{m, n, \lambda}(0):=B_{m, n, \lambda}$ called the degenerate truncated Bernoulli numbers provided by

$$
\sum_{n=0}^{\infty} B_{m, n, \lambda} \frac{z^{n}}{n !}=\frac{(1)_{m+1, \lambda} \frac{z^{m+1}}{(m+1) !}}{e_{\lambda}(z)-1-e_{m-1, \lambda}(z)} .
$$

The polynomials $B_{m, n, \lambda}(x)$ in conjuction with the several identities and formulas are analyzed in [8] with details. We now introduce the Gould-Hopper based degenerate truncated Bernoulli polynomials as follows.

Definition 2.1. Let $x$ and $y$ be two independent variables and $j \in \mathbb{N}_{0}$. The Gould-Hopper based degenerate truncated Bernoulli polynomials are defined below:

$$
\sum_{n=0}^{\infty}{ }_{H} B_{m, n, \lambda}^{(j)}(x, y) \frac{z^{n}}{n !}=\frac{(1)_{m+1, \lambda} \frac{z^{m+1}}{(m+1) !}}{e_{\lambda}(z)-1-e_{m-1, \lambda}(z)} e_{\lambda}^{x}(z) e_{\lambda}^{y}\left(z^{j}\right) .
$$

We choose to call the Gould-Hopper based Detr-Bernoulli polynomials instead of the Gould-Hopper based degenerate truncated Bernoulli polynomials.

Remark 2.1. When $x=0$ in Definition 2.1, the Gould-Hopper based Detr-Bernoulli polynomials $B_{m, n, \lambda}(x)$ reduce to the following polynomials which is also new extension of the Detr-Bernoulli polynomials:

$$
\sum_{n=0}^{\infty} B_{m, n, \lambda}^{(j)}(y) \frac{z^{n}}{n !}=\frac{(1)_{m+1, \lambda} \frac{z^{m+1}}{(m+1) !}}{e_{\lambda}(z)-1-e_{m-1, \lambda}(z)} e_{\lambda}^{y}\left(z^{j}\right) .
$$

Remark 2.2. Taking $x=y=0$ in Definition 2.1, the polynomials ${ }_{H} B_{m, n, \lambda}^{(j)}(x, y)$ reduce to the degenerate truncated Bernoulli numbers in (2.3).

Theorem 2.1. The following summation formulae holds for $n \in \mathbb{N}_{0}$ :

$$
{ }_{H} B_{m, n, \lambda}^{(j)}(x, y)=\sum_{k=0}^{n}\left(\begin{array}{l}
n \\
k
\end{array}\right)(x)_{k, \lambda H} B_{m, n-k, \lambda}^{(j)}(y)
$$

and

$$
{ }_{H} B_{m, n, \lambda}^{(j)}(x, y)=\sum_{k=0}^{n}\left(\begin{array}{l}
n \\
k
\end{array}\right) H_{n-k, \lambda}^{(j)}(x, y) B_{m, k, \lambda} .
$$


Proof. By Definition 2.1 and utilizing the (2.5) and (2.3), we attain

$$
\begin{aligned}
\sum_{n=0}^{\infty}{ }_{H} B_{m, n, \lambda}^{(j)}(x, y) \frac{z^{n}}{n !} & =\frac{(1)_{m+1, \lambda} \frac{z^{m+1}}{(m+1) !}}{e_{\lambda}(z)-1-e_{m-1, \lambda}(z)} e_{\lambda}^{x}(z) e_{\lambda}^{y}\left(z^{j}\right) \\
& =\sum_{n=0}^{\infty}{ }_{H} B_{m, n, \lambda}^{(j)}(y) \frac{z^{n}}{n !} \sum_{n=0}^{\infty}(x)_{n, \lambda} \frac{z^{n}}{n !} \\
& =\sum_{n=0}^{\infty}\left(\sum_{k=0}^{n}\left(\begin{array}{l}
n \\
k
\end{array}\right)(x)_{k, \lambda} H B_{m, n-k, \lambda}^{(j)}(y)\right) \frac{z^{n}}{n !}
\end{aligned}
$$

and

$$
\begin{aligned}
\sum_{n=0}^{\infty}{ }_{H} B_{m, n, \lambda}^{(j)}(x, y) \frac{z^{n}}{n !} & =\frac{(1)_{m+1, \lambda} \frac{z^{m+1}}{(m+1) !}}{e_{\lambda}(z)-1-e_{m-1, \lambda}(z)} e_{\lambda}^{x}(z) e_{\lambda}^{y}\left(z^{j}\right) \\
& =\sum_{n=0}^{\infty} H_{n, \lambda}^{(j)}(x, y) \frac{z^{n}}{n !} \sum_{n=0}^{\infty} B_{m, n, \lambda} \frac{z^{n}}{n !} \\
& =\sum_{n=0}^{\infty}\left(\sum_{k=0}^{n}\left(\begin{array}{l}
n \\
k
\end{array}\right) H_{n-k, \lambda}^{(j)}(x, y) B_{m, k, \lambda}\right) \frac{z^{n}}{n !}
\end{aligned}
$$

which complete the proof.

We give the following lemma.

Lemma 2.1. (cf. [6]) The following series manipulation is valid:

$$
\sum_{n=0}^{\infty} \sum_{k=0}^{\infty} A(k, n)=\sum_{n=0}^{\infty} \sum_{k=0}^{\lfloor n / j\rfloor} A(k, n-j k),
$$

where $L \cdot\rfloor$ is the Gauss symbol, and shows the maximum integer that does not exceed the number in the square brackets.

We give the following theorem.

Theorem 2.2. We have

$$
{ }_{H} B_{m, n, \lambda}^{(j)}(x, y)=n ! \sum_{k=0}^{\lfloor n / j\rfloor} \frac{(y)_{n-j k, \lambda}}{k !(n-j k) !} B_{m, k, \lambda}(x) .
$$

Proof. By applying (2.7) and using the following equality

$$
\begin{aligned}
\sum_{n=0}^{\infty}{ }_{H} B_{m, n, \lambda}^{(j)}(x, y) \frac{z^{n}}{n !} & =\frac{(1)_{m+1, \lambda} \frac{z^{m+1}}{(m+1) !}}{e_{\lambda}(z)-1-e_{m-1, \lambda}(z)} e_{\lambda}^{x}(z) e_{\lambda}^{y}\left(z^{j}\right) \\
& =\left(\sum_{n=0}^{\infty} B_{m, n, \lambda}(x) \frac{z^{n}}{n !}\right)\left(\sum_{n=0}^{\infty}(y)_{n, \lambda} \frac{z^{j n}}{n !}\right) \\
& =\sum_{n=0}^{\infty}\left(n ! \sum_{k=0}^{\lfloor n / j\rfloor} \frac{(y)_{n-j k, \lambda}}{k !(n-j k) !} B_{m, k, \lambda}(x)\right) \frac{z^{n}}{n !},
\end{aligned}
$$

which is the claimed result (2.8).

Theorem 2.3. We have

$$
{ }_{H} B_{m, n, \lambda}^{(j)}\left(x_{1}+x_{2}, y_{1}+y_{2}\right)=\sum_{k=0}^{n}\left(\begin{array}{l}
n \\
k
\end{array}\right){ }_{H} B_{m, k, \lambda}^{(j)}\left(x_{1}, y_{1}\right) H_{n-k, \lambda}^{(j)}\left(x_{2}, y_{2}\right) .
$$


Proof. Using the following equality

$$
\frac{(1)_{m+1, \lambda} \frac{z^{m+1}}{(m+1) !}}{e_{\lambda}(z)-1-e_{m-1, \lambda}(z)} e_{\lambda}^{x_{1}+x_{2}}(z) e_{\lambda}^{y_{1}+y_{2}}\left(z^{j}\right)=\frac{(1)_{m+1, \lambda} \frac{z^{m+1}}{(m+1) !}}{e_{\lambda}(z)-1-e_{m-1, \lambda}(z)} e_{\lambda}^{x_{1}}(z) e_{\lambda}^{y_{1}}\left(z^{j}\right) e_{\lambda}^{x_{2}}(z) e_{\lambda}^{y_{2}}\left(z^{j}\right),
$$

the proof is similar to Theorem 2.1. We, therefore, choose to omit details involved.

Theorem 2.4. We have

$$
\frac{\partial}{\partial x}{ }_{H} B_{m, n, \lambda}^{(j)}(x, y)=n ! \sum_{s=1}^{\infty}{ }_{H} B_{m, n-s, \lambda}^{(j)}(x, y) \frac{(-1)^{s+1}}{(n-s) ! s} \lambda^{s-1} .
$$

Proof. By appliying the operator $\frac{\partial}{\partial x}$ to both sides of (2.4), we then derive

$$
\begin{aligned}
\sum_{n=0}^{\infty} \frac{\partial}{\partial x}{ }_{H} B_{m, n, \lambda}^{(j)}(x, y) \frac{z^{n}}{n !} & =\frac{(1)_{m+1, \lambda} \frac{z^{m+1}}{(m+1) !} e_{\lambda}^{y}\left(z^{j}\right)}{e_{\lambda}(z)-1-e_{m-1, \lambda}(z)} \frac{\partial}{\partial x}(1+\lambda z)^{\frac{x}{\lambda}} \\
& =\frac{(1)_{m+1, \lambda} \frac{z^{m+1}}{(m+1) !} e_{\lambda}^{y}\left(z^{j}\right)}{e_{\lambda}(z)-1-e_{m-1, \lambda}(z)}(1+\lambda z)^{\frac{x}{\lambda}} \ln (1+\lambda z)^{\frac{1}{\lambda}} \\
& =\sum_{n=0}^{\infty}{ }_{H} B_{m, n, \lambda}^{(j)}(x, y) \frac{z^{n}}{n !} \sum_{s=1}^{\infty} \frac{(-1)^{s+1}}{s} \lambda^{s-1} z^{s} \\
& =\sum_{n=0}^{\infty} \sum_{s=1}^{\infty} H B_{m, n, \lambda}^{(j)}(x, y) \frac{(-1)^{s+1}}{s} \lambda^{s-1} \frac{z^{n+s}}{n !},
\end{aligned}
$$

which means the assertion in (2.10).

Theorem 2.5. For $n, m \in \mathbb{N}_{0}$, we have

$$
\begin{aligned}
{ }_{H} B_{m+1, n, \lambda}^{(j)}(x, y)= & n \frac{1-(m+1) \lambda}{m+2}{ }_{H} B_{m, n-1, \lambda}^{(j)}(x, y) \\
& +\frac{(m+1) !}{(1-m \lambda)} \sum_{k=0}^{n}\left(\begin{array}{c}
n+1 \\
k
\end{array}\right) B_{m, k ; \lambda H} B_{m+1, n+1-k, \lambda}^{(j)}(x, y) .
\end{aligned}
$$

Proof. Utilizing the following equality

$$
\begin{gathered}
(1)_{m+2, \lambda} \frac{z^{m+2}}{(m+2) !} e_{\lambda}^{x}(z) e_{\lambda}^{y}\left(z^{j}\right)=\left(e_{\lambda}(z)-1-e_{m, \lambda}(z)\right) \sum_{n=0}^{\infty}{ }_{H} B_{m+1, n, \lambda}^{(j)}(x, y) \frac{z^{n}}{n !} \\
=\left(e_{\lambda}(z)-1-e_{m-1, \lambda}(z)\right) \sum_{n=0}^{\infty}{ }_{H} B_{m+1, n, \lambda}^{(j)}(x, y) \frac{z^{n}}{n !} \\
-(1)_{m, \lambda} \frac{z^{m}}{m !} \sum_{n=0}^{\infty}{ }_{H} B_{m+1, n, \lambda}^{(j)}(x, y) \frac{z^{n}}{n !},
\end{gathered}
$$

the proof is similar to Theorem 2.1. We, therefore, choose to omit details involved.

Theorem 2.6. For $n, m \in \mathbb{N}_{0}$, we have

$$
\frac{(1)_{m+1, \lambda}}{(m+1) !} H_{n, \lambda}^{(j)}(x, y)=\sum_{k=0}^{n+1} n !(1)_{k+m, \lambda} \frac{{ }_{H} B_{m, n+1-k, \lambda}^{(j)}(x, y)}{(k+m) !(n+1-k) !}-n ! \frac{{ }_{H} B_{m, n+m+1, \lambda}^{(j)}(x, y)}{(n+m+1) !} .
$$

Proof. By Definition 2.1, we have

$$
\begin{aligned}
(1)_{m+1, \lambda} \frac{z^{m+1}}{(m+1) !} e_{\lambda}^{x}(z) e_{\lambda}^{y}\left(z^{j}\right) & =\left(e_{\lambda}(z)-1-e_{m-1, \lambda}(z)\right) \sum_{n=0}^{\infty}{ }_{H} B_{m, n, \lambda}^{(j)}(x, y) \frac{z^{n}}{n !} \\
& =\sum_{n=m}^{\infty}(1)_{n, \lambda} \frac{z^{n}}{n !} \sum_{n=0}^{\infty}{ }_{H} B_{m, n, \lambda}^{(j)}(x, y) \frac{z^{n}}{n !}-\sum_{n=0}^{\infty}{ }_{H} B_{m, n, \lambda}^{(j)}(x, y) \frac{z^{n}}{n !},
\end{aligned}
$$

which yields the asserted result (2.12). 
In recent years, many mathematicians have been studied special polynomials to acquire some of their symmetric identities and implicit summation formulas, $c f$. $[5,15]$ and see also each of the references cited therein. We now derive some the mentioned formulas and identities for the polynomials ${ }_{H} B_{m, n, \lambda}^{(j)}(x, y)$.

Theorem 2.7. For $n, m \in \mathbb{N}_{0}$, we have

$$
{ }_{H} B_{m, n, \lambda}^{(j)}(x, y)=\sum_{l=0}^{n} \sum_{k=0}^{n}\left(\begin{array}{l}
n \\
k
\end{array}\right) B_{m, n-k, \lambda}^{(j)}(y) S_{2 ; \lambda}(k, l:-l)(x)^{(l)},
$$

where $(x)^{(l)}=x(x+1)(x+2) \cdots(x+(l-1))$ for $l \in \mathbb{N}$ with $(x)^{(l)}:=1$ (cf. [8]).

Proof. From Definition 2.1 and utilizing (1.6) and (2.5), we acquire

$$
\begin{aligned}
\sum_{n=0}^{\infty}{ }_{H} B_{m, n, \lambda}^{(j)}(x, y) \frac{z^{n}}{n !} & =\frac{(1)_{m+1, \lambda} \frac{z^{m+1}}{(m+1) !} e_{\lambda}^{y}\left(z^{j}\right)}{e_{\lambda}(z)-1-e_{m-1, \lambda}(z)}\left(e_{\lambda}^{-1}(z)-1+1\right)^{x} \\
& =\frac{(1)_{m+1, \lambda} \frac{z^{m+1}}{(m+1) !} e_{\lambda}^{y}\left(z^{j}\right)}{e_{\lambda}(z)-1-e_{m-1, \lambda}(z)} \sum_{l=0}^{\infty}\left(\begin{array}{c}
x+l-1 \\
l
\end{array}\right)\left(1-e_{\lambda}^{-1}(z)\right)^{l} \\
& =\frac{(1)_{m+1, \lambda} \frac{z^{m+1}}{(m+1) !} e_{\lambda}^{y}\left(z^{j}\right)}{e_{\lambda}(z)-1-e_{m-1, \lambda}(z)} \sum_{l=0}^{\infty}\left(\begin{array}{c}
x+l-1 \\
l
\end{array}\right) \frac{\left(e_{\lambda}(z)-1\right)^{l}}{l !} e_{\lambda}^{-l}(z) l ! \\
& =\sum_{l=0}^{\infty}(x)^{(l)} \sum_{n=0}^{\infty} B_{m, n, \lambda}^{(j)}(y) \frac{z^{n}}{n !} \sum_{n=0}^{\infty} S_{2 ; \lambda}(n, l:-l) \frac{z^{n}}{n !} \\
& =\sum_{l=0}^{\infty}(x)^{(l)} \sum_{n=0}^{\infty}\left(\sum_{k=0}^{n}\left(\begin{array}{l}
n \\
k
\end{array}\right) B_{m, n-k, \lambda}^{(j)}(y) S_{2 ; \lambda}(k, l:-l)\right) \frac{z^{n}}{n !},
\end{aligned}
$$

which means the assertion (2.13).

Note that $(c f .[5,15])$

$$
\sum_{N=0}^{\infty} f(N) \frac{(x+y)^{N}}{N !}=\sum_{n, s=0}^{\infty} f(n+s) \frac{x^{n}}{n !} \frac{y^{s}}{s !} .
$$

We give the following theorem.

Theorem 2.8. We have

$$
{ }_{H} B_{m, k+l, \lambda}^{(j)}(x, y)=\sum_{n, s=0}^{k, l}\left(\begin{array}{l}
k \\
n
\end{array}\right)\left(\begin{array}{l}
l \\
s
\end{array}\right)(\mu-x)_{n+s, \lambda} H_{m, k+l-n-s, \lambda}^{(j)}(\mu, y) .
$$

Proof. Taking $z$ by $z+\omega$ in (2.4), it yields

$$
\frac{(1)_{m+1, \lambda} \frac{(z+\omega)^{m+1}}{(m+1) !}}{e_{\lambda}(z+\omega)-1-e_{m-1, \lambda}(z+\omega)} e_{\lambda}^{y}\left((z+\omega)^{j}\right)=e_{\lambda}^{\mu}(z+\omega) \sum_{k, l=0}^{\infty}{ }_{H} B_{m, k+l, \lambda}^{(j)}(\mu, y) \frac{z^{k}}{k !} \frac{\omega^{l}}{l !}
$$

and similarly we acquire

$$
\frac{(1)_{m+1, \lambda} \frac{(z+\omega)^{m+1}}{(m+1) !}}{e_{\lambda}(z+\omega)-1-e_{m-1, \lambda}(z+\omega)} e_{\lambda}^{y}\left((z+\omega)^{j}\right)=e_{\lambda}^{x}(z+\omega) \sum_{k, l=0}^{\infty}{ }_{H} B_{m, k+l, \lambda}^{(j)}(x, y) \frac{z^{k}}{k !} \frac{\omega^{l}}{l !} .
$$

By the last two equalities, we write

$$
\begin{aligned}
\sum_{k, l=0}^{\infty}{ }_{H} B_{m, k+l, \lambda}^{(j)}(x, y) \frac{z^{k}}{k !} \frac{\omega^{l}}{l !} & =e_{\lambda}^{\mu-x}(z+\omega) \sum_{k, l=0}^{\infty}{ }_{H} B_{m, k+l, \lambda}^{(j)}(\mu, y) \frac{z^{k}}{k !} \frac{\omega^{l}}{l !} \\
& =\sum_{n, s=0}^{\infty}(\mu-x)_{n+s, \lambda} \frac{z^{n}}{n !} \frac{\omega^{m}}{s !} \sum_{k, l=0}^{\infty}{ }_{H} B_{m, k+l, \lambda}^{(j)}(\mu, y) \frac{z^{k}}{k !} \frac{\omega^{l}}{l !}
\end{aligned}
$$


By using (2.14), we acquire

$$
\sum_{k, l=0}^{\infty}{ }_{H} B_{m, k+l, \lambda}^{(j)}(x, y) \frac{z^{k}}{k !} \frac{\omega^{l}}{l !}=\sum_{k, l=0}^{\infty} \sum_{n, s=0}^{k, l} \frac{(\mu-x)_{n+s, \lambda} B_{m, k+l-n-s, \lambda}^{(j)}(\mu, y)}{n ! s !(k-l) !(l-s) !} z^{k} \omega^{l},
$$

whichmeans the assertion (2.15).

Theorem 2.9. The following symmetric identity holds for $n \in \mathbb{N}_{0}$ and $a, b \in \mathbb{R}$ :

$$
\sum_{k=0}^{n}\left(\begin{array}{l}
n \\
k
\end{array}\right){ }_{H} B_{m, n-k, \lambda}^{(j)}(b x, y){ }_{H} B_{m, k, \lambda}^{(j)}(a x, y) a^{n-k} b^{k}=\sum_{k=0}^{n}\left(\begin{array}{l}
n \\
k
\end{array}\right){ }_{H} B_{m, n-k, \lambda}^{(j)}(a x, y)_{H} B_{m, k, \lambda}^{(j)}(b x, y) b^{n-k} a^{k}
$$

Proof. Let

$$
\Upsilon=\frac{(a z)^{m+1}(b z)^{m+1}\left(\frac{(1)_{m+1, \lambda}}{(m+1) !}\right)^{2} e_{\lambda}^{b x}(a z) e_{\lambda}^{a x}(b z) e_{\lambda}^{y}\left(a^{j} z^{j}\right) e_{\lambda}^{y}\left(b^{j} z^{j}\right)}{\left(e_{\lambda}(a z)-1-e_{m-1, \lambda}(a z)\right)\left(e_{\lambda}(b z)-1-e_{m-1, \lambda}(b z)\right)}
$$

Then, thanks to $\Upsilon$ being symmetric in $a$ and $b$, we have two expansions of $\Upsilon$ as follows:

$$
\begin{aligned}
\Upsilon & =\sum_{n=0}^{\infty}{ }_{H} B_{m, n, \lambda}^{(j)}(b x, y) \frac{(a z)^{n}}{n !} \sum_{n=0}^{\infty}{ }_{H} B_{m, n, \lambda}^{(j)}(a x, y) \frac{(b z)^{n}}{n !} \\
& =\sum_{n=0}^{\infty} \sum_{k=0}^{n}\left(\begin{array}{l}
n \\
k
\end{array}\right){ }_{H} B_{m, n-k, \lambda}^{(j)}(b x, y)_{H} B_{m, k, \lambda}^{(j)}(a x, y) a^{n-k} b^{k} \frac{z^{n}}{n !}
\end{aligned}
$$

and similarly

$$
\Upsilon=\sum_{n=0}^{\infty} \sum_{k=0}^{n}\left(\begin{array}{l}
n \\
k
\end{array}\right){ }_{H} B_{m, n-k, \lambda}^{(j)}(a x, y){ }_{H} B_{m, k, \lambda}^{(j)}(b x, y) b^{n-k} a^{k} \frac{z^{n}}{n !},
$$

which means the assertion (2.16).

For $k, m \in \mathbb{N}_{0}$, we define the numbers $S_{m, k, \lambda}(n)$ as follows:

$$
\frac{e_{\lambda}((n+1) z)-1-e_{m-1, \lambda}((n+1) z)}{e_{\lambda}(z)-1-e_{m-1, \lambda}(z)}=\sum_{k=0}^{\infty} S_{m, k, \lambda}(n) \frac{z^{k}}{k !},
$$

which, for $\lambda \rightarrow m=0$, reduces to the power sum $\lim _{\lambda \rightarrow 0} S_{0, k, \lambda}(n):=S_{k}(n)$ given by (cf. [5])

$$
\sum_{k=0}^{\infty} S_{k}(n) \frac{z^{k}}{k !}=\frac{e^{(n+1) z}-1}{e^{z}-1} .
$$

A symmetric identity for ${ }_{H} B_{m, n, \lambda}^{(j)}(x, y)$ is stated below.

Theorem 2.10. For $a, b$ being two integers and $n, m \in \mathbb{N}_{0}$, we have

$$
\begin{aligned}
& \sum_{u=0}^{n} \sum_{j=0}^{n-u} \sum_{i=0}^{u}\left(\begin{array}{l}
n \\
u
\end{array}\right)\left(\begin{array}{c}
n-u \\
j
\end{array}\right)\left(\begin{array}{c}
n \\
i
\end{array}\right){ }_{H} B_{m, n-u-j, \lambda}^{(j)}(b x, b y) S_{m, j, \lambda}(b-1) \\
& \times{ }_{H} B_{m, u-i, \lambda}^{(j)}(a x, a y) S_{m, i, \lambda}(a-1) a^{n-u} b^{u} \\
&=\sum_{u=0}^{n} \sum_{j=0}^{n-u} \sum_{i=0}^{u}\left(\begin{array}{c}
n \\
u
\end{array}\right)\left(\begin{array}{c}
n-u \\
j
\end{array}\right)\left(\begin{array}{c}
n \\
i
\end{array}\right)_{H} B_{m, n-u-j, \lambda}^{(j)}(a x, a y) S_{m, j, \lambda}(a-1) \\
&{ }_{H} B_{m, u-i, \lambda}^{(j)}(b x, b y) S_{m, i, \lambda}(b-1) b^{n-u} a^{u} .
\end{aligned}
$$


Proof. Let

$$
\begin{aligned}
\Psi= & \frac{(a z)^{m+1}(b z)^{m+1}\left(\frac{(1)_{m+1, \lambda}}{(m+1) !}\right)^{2}\left(e_{\lambda}(a b z)-1-e_{m-1, \lambda}(a b z)\right)^{2}}{\left(e_{\lambda}(a z)-1-e_{m-1, \lambda}(a z)\right)^{2}\left(e_{\lambda}(b z)-1-e_{m-1, \lambda}(b z)\right)^{2}} \\
& \times e_{\lambda}^{b x}(a z) e_{\lambda}^{a x}(b z) e_{\lambda}^{b y}\left(a^{j} z^{j}\right) e_{\lambda}^{a y}\left(b^{j} z^{j}\right) \\
= & \frac{(a z)^{m+1} \frac{(1)_{m+1, \lambda}}{(m+1) !} e_{\lambda}^{b x}(a z) e_{\lambda}^{b y}\left(a^{j} z^{j}\right)}{e_{\lambda}(a z)-1-e_{m-1, \lambda}(a z)} \frac{e_{\lambda}(a b z)-1-e_{m-1, \lambda}(a b z)}{e_{\lambda}(a z)-1-e_{m-1, \lambda}(a z)} \\
& \times \frac{(b z)^{m+1} \frac{(1)_{m+1, \lambda}}{(m+1) !} e_{\lambda}^{a x}(b z) e_{\lambda}^{a y}\left(b^{j} z^{j}\right)}{e_{\lambda}(b z)-1-e_{m-1, \lambda}(b z)} \frac{e_{\lambda}(a b z)-1-e_{m-1, \lambda}(a b z)}{e_{\lambda}(b z)-1-e_{m-1, \lambda}(b z)}
\end{aligned}
$$

Thus we have

$$
\begin{aligned}
\Psi= & \sum_{n=0}^{\infty} \sum_{l=0}^{n}\left(\begin{array}{l}
n \\
l
\end{array}\right){ }_{H} B_{m, n-l, \lambda}^{(j)}(b x, b y) S_{m, l, \lambda}(b-1) \frac{(a z)^{n}}{n !} \\
& \times \sum_{n=0}^{\infty} \sum_{l=0}^{n}\left(\begin{array}{c}
n \\
l
\end{array}\right){ }_{H} B_{m, n-l, \lambda}^{(j)}(a x, a y) S_{m, l, \lambda}(a-1) \frac{(b z)^{n}}{n !}
\end{aligned}
$$

which completes the proof.

\section{Further Remarks}

Now, we introduce the Gould-Hopper based Detr-Bernoulli polynomials $B_{m, n, \lambda}^{(r)}(x)$ of order $r$ as follows:

$$
\sum_{n=0}^{\infty}{ }_{H} B_{m, n, \lambda}^{(j, r)}(x, y) \frac{z^{n}}{n !}=\left(\frac{(1)_{m+1, \lambda} \frac{z^{m+1}}{(m+1) !}}{e_{\lambda}(z)-1-e_{m-1, \lambda}(z)}\right)^{r} e_{\lambda}^{x}(z) e_{\lambda}^{y}\left(z^{j}\right) .
$$

We note that ${ }_{H} B_{m, n, \lambda}^{(j, 1)}(x, y):={ }_{H} B_{m, n, \lambda}^{(j)}(x, y)$. Also, upon letting $x=y=0$, the polynomials in (3.1) reduce to the Gould-Hopper based Detr-Bernoulli numbers of order $r$ below:

$$
\sum_{n=0}^{\infty} B_{m, n, \lambda}^{(r)} \frac{z^{n}}{n !}=\left(\frac{(1)_{m+1, \lambda} \frac{z^{m+1}}{(m+1) !}}{e_{\lambda}(z)-1-e_{m-1, \lambda}(z)}\right)^{r} .
$$

We first give the following summation formula.

Theorem 3.1. We have

$$
{ }_{H} B_{m, n, \lambda}^{(j, r)}(x, y)=\sum_{l=0}^{n}\left(\begin{array}{l}
n \\
l
\end{array}\right) B_{m, n-l, \lambda}^{(r)} H_{l, \lambda}^{(j)}(x, y)
$$

Proof. By using (1.8) and (3.1), the proof is similar to Theorem 2.1. We, therefore, choose to omit details involved.

Addition property of the Gould-Hopper based Detr-Bernoulli polynomials of order $r$ is given below.

Theorem 3.2. We have

$$
{ }_{H} B_{m, n, \lambda}^{\left(j, r_{1}+r_{2}\right)}\left(x_{1}+x_{2}, y_{1}+y_{2}\right)=\sum_{u=0}^{n}\left(\begin{array}{l}
n \\
u
\end{array}\right){ }_{H} B_{m, u, \lambda}^{\left(j, r_{1}\right)}\left(x_{1}, y_{1}\right)_{H} B_{m, n-u, \lambda}^{\left(j, r_{2}\right)}\left(x_{2}, y_{2}\right) .
$$

Proof. By utlizing (1.8) and (3.1), the proof is similar to Theorem 2.1. We, therefore, choose to omit details involved. 
Theorem 3.3. We have

$$
\begin{gathered}
H_{n, \lambda}^{(j)}(x+\mu, y)=\frac{n ! r !\left((1)_{m+1, \lambda}\right)^{r}}{(n+(m+1)) !((m+1) !)^{r}} \\
\times \sum_{l=0}^{n+(m+1) r}\left(\begin{array}{c}
n+(m+1) \\
l
\end{array}\right) S_{2, m ; \lambda}(n+(m+1)-l, r: \mu)_{H} B_{m, l, \lambda}^{(j, r)}(x, y) .
\end{gathered}
$$

Proof. By (1.7), (1.8) and (3.1), we investigate

$$
\begin{aligned}
\sum_{n=0}^{\infty} S_{2, m ; \lambda}(n, r: \mu) \frac{z^{n}}{n !} \sum_{n=0}^{\infty} H_{H, n, \lambda} B_{m, r)}^{(j, x)}(x, y) & =\frac{\left(z_{\lambda}(z)-1-e_{m-1, \lambda}(z)\right)^{r}}{r !} e_{\lambda}^{\mu}(z) \\
& \times \frac{\left((1)_{m+1, \lambda}\right)^{r} \frac{z^{(m+1) r}}{((m+1) !)^{r}}}{\left(e_{\lambda}(z)-1-e_{m-1, \lambda}(z)\right)^{r}} e_{\lambda}^{x}(z) e_{\lambda}^{y}\left(z^{j}\right) \\
= & e_{\lambda}^{x+\mu}(z) e_{\lambda}^{y}\left(z^{j}\right) \frac{\left((1)_{m+1, \lambda}\right)^{r}}{r !} \frac{z^{(m+1) r}}{((m+1) !)^{r}} \\
= & \sum_{n=0}^{\infty} H_{n, \lambda}^{(j)}(x+\mu, y) \frac{z^{n+(m+1) r}}{n !} \frac{\left((1)_{m+1, \lambda}\right)^{r}}{r !((m+1) !)^{r}},
\end{aligned}
$$

which implies the assertion (3.2).

\section{Conclusion}

In this study, we have introduced the Gould-Hopper based truncated degenerate Bernoulli polynomials and have examined diverse properties and formulas covering addition formulas, derivation rule and relationships with the Gould-Hopper polynomials and the degenerate Stirling numbers of the second. Then, we have derived some interesting symmetric relations and implicit summation identities. Moreover, we have defined Gould-Hopper based truncated degenerate Bernoulli polynomials of order $r$ and have given some of their properties and relations.

\section{Acknowledgment.}

We thank the reviewer for their insightful comments and suggestions that helped us improve the paper.

\section{Funding}

There is no funding for this work.

\section{Availability of data and materials}

Not applicable.

\section{Competing interests}

The authors declare that they have no competing interests.

\section{Author's contributions}

All authors contributed equally to the writing of this paper. All authors read and approved the final manuscript. 


\section{References}

[1] Carlitz, L.: Degenerate Stirling, Bernoulli and Eulerian numbers. Utilitas Mathematica. 15, 51-88 (1975).

[2] Cheon,G.-S.: A note on the Bernoulli and Euler polynomials. Applied Mathematics Letters. 16, 365-368 (2003).

[3] Dattoli, G., Ceserano, C., Sacchetti, D.: A note on truncated polynomials. Applied Mathematics and Computation. 134, 595-605 (2003).

[4] Duran, U, Acikgoz, M.: Generalized Gould-Hopper based fully degenerate central Bell polynomials. Turkish Journal of Analysis and Number Theory. 7 (5), 124-134 (2019).

[5] Duran, U., Araci, S., Acikgoz, M.: Bell-Based Bernoulli polynomials with applications. Axioms. 10, no. 29 (2021).

[6] Duran, U., Sadjang, P.N.: On Gould-Hopper-based fully degenerate poly-Bernoulli polynomials with a q-parameter. Mathematics. 7, no. 121 (2019).

[7] Duran, U., Acikgoz, M.: On generalized degenerate Gould-Hopper based fully degenerate Bell polynomials. Journal of Mathematics and Computer Science. 21 (3), 243-257 (2020).

[8] Duran, U., Acikgoz, M.: On Degenerate Truncated Special Polynomials. Mathematics. 8 (1), no. 144 (2020).

[9] Hassen, A., Nguyen, H.D.: Hypergeometric Bernoulli polynomials and Appell sequences. International Journal of Number Theory. 4, 767-774 (2008).

[10] Howard, F.T.: Explicit formulas for degenerate Bernoulli numbers. Discrete Mathematics. 162, 175-185 (1996).

[11] Kim, T., Kim, D.S., Jang, L.-C., Kwon, H.I.: Extended degenerate stirling numbers of the second kind and extended degenerate Bell polynomials. Utilitas Mathematica. 106, 11-21 (2018).

[12] Kim, T., Yao, Y., Kim, D.S., Jang, G.-W.: Degenerate r-Stirling numbers and r-Bell polynomials. Russian Journal of Mathematical Physics. 25, 44-58 (2018).

[13] Kim, T.: A note on degenerate Stirling polynomials of the second kind. Proceedings of the Jangjeon Mathematical Society. 20, 319-331 (2017).

[14] Kurt, B: Explicit relations for the modified degenerate Apostol-type polynomials. Journal of Balikesir University Institute of Science and Technology. 20, 401-412 (2018).

[15] Pathan, M.A., Khan, W.A.: Some implicit summation formulas and symmetric identities for the generalized HermiteBernoulli polynomials. Mediterranean Journal of Mathematics. 12, 679-695 (2015).

[16] Srivastava, H.M., Araci, S., Khan, W.A., Acikgoz, M.: A note on the truncated-exponential based Apostol-type polynomials. Symmetry. 11, no. 538 (2019).

[17] Srivastava, H.M., Choi, J.: Zeta and $q$-Zeta functions and associated series and integrals. Elsevier Science Publishers. Amsterdam (2012).

[18] Srivastava, H.M., Pinter, A.: Remarks on some relationships between the Bernoulli and Euler polynomials. Applied Mathematics Letters. 17, 375-380 (2004).

\section{Affiliation}

\section{UĞUR DURAN}

ADDRESS: Iskenderun Technical University, Faculty of Engineering and Natural Sciences, Department of the Basic Concepts of Engineering, TR-31200 Hatay,Turkey.

E-MAIL: mtdrnugur@gmail.com \& ugur.duran@iste.edu.tr

ORCID ID: 0000-0002-5717-1199 